\title{
Agriostomum vryburgi Railliet, 1902, parasite nouveau des bovidés domestiques de la République Populaire du Congo
}

\author{
par M. GRABER $\left({ }^{*}\right)$ et M. TURPIN $\left({ }^{* *}\right)$
}

\begin{abstract}
RÉSUMÉ
Les auteurs signalent la prèsence pour la première fois en Afrique, d'Agriostomes de l'espèce Agriostomum vryburgi Railliet, 1902, dans le tractus digestif de bovins N'Dama importés du Sénégal.

La possibilité de l'introduction de ce parasite dans la Républıque Popularre du Congo par du bétail importé est discutée.
\end{abstract}

Au cours d'une enquête portant sur le parasitisme interne des bovins N'Dama élevés sur le Ranch de la Louila (Vallée du Niari -- République populaire du Congo), quelques animaux ont été sacrifiés et autopsiés (11). Ont été découverts :

- dans la panse, un Gastrothylacidae, Carmyerius spatiosus Brandes, 1898 ;

- dans les veines mésentériques, un Schistosomidae, Schistosoma bovis Sonsino, 1876.

- dans le côlon et le caecum, un Strongylidae, Oesophagostomum (Bosicola) radiatum avec des lésions massives d'œsophagostomose nodulaire.

Dans le lot d'Oesophagostomes adultes, ont été isolés, dans la proportion de 1 pour 7, des Nématodes appartenant au genre Agriostomum, groupe intermédiaire entre les Ancylostominae et les Oesophagostominae et à l'espèce Agriostomum vryburgi Railliet, 1902.

C'est la première fois que la présence de ce parasite, connu depuis fort longtemps déjà dans d'autres pays du monde, est signalée sur le continent africain. De ce fait, il mérite de retenir un peu plus l'attention.

(*) Ecole Nationale Vétérinaire, Chaire de Parasitologie, 2, Quai Chauveau, 69337 Lyon Cedex 1.

(**) COGLA, B. P. 45, 92360 Meudon-Ja-Forêt.

\section{DESCRIPTION}

Les descriptions antérieures $(5,15,17,19,25)$ étant, pour la plupart, insuffisantes, il a paru utile de redécrire entièrement le Nématode.

Le corps est cylindrique, blanc-grisâtre, effilé aux deux extrémités. L'antérieure est recourbée sur la face dorsale, ce que l'on observe facilement à l'cil nu.

La cuticule est finement striée et l'intervalle entre chaque strie est compris entre 2 et $2,5 \mu$. Bien que, dans la région céphalique, en avant de l'ouverture de la cavité buccale, elle forme une sorte de petit capuchon plus ou moins marqué, il n'existe pas, à proprement parler, de renflement vésiculaire (la « vésicule céphalique » de certains Oesophagostomes). Par contre, on observe, dans tous les cas, une fente ventrale transversale située, en moyenne, à $438 \mu$ de l'apex (400-480 $\mu$ ).

La tête porte 4 papilles submédianes proéminentes et 2 amphides latérales. Elle s'ouvre dorsalement. Elle est soutenue par un cercle chitineux pourvu de dents ou odontia (fig. 1, 2), ̀̀ raison de deux ventrales et, de chaque côté, une paire de subventrales, une paire de latérales et une paire de subdorsales, soit, au total, 14 dents, ce qui confirme la définition du genre Agriostomum donnée par MONNIG (15). 

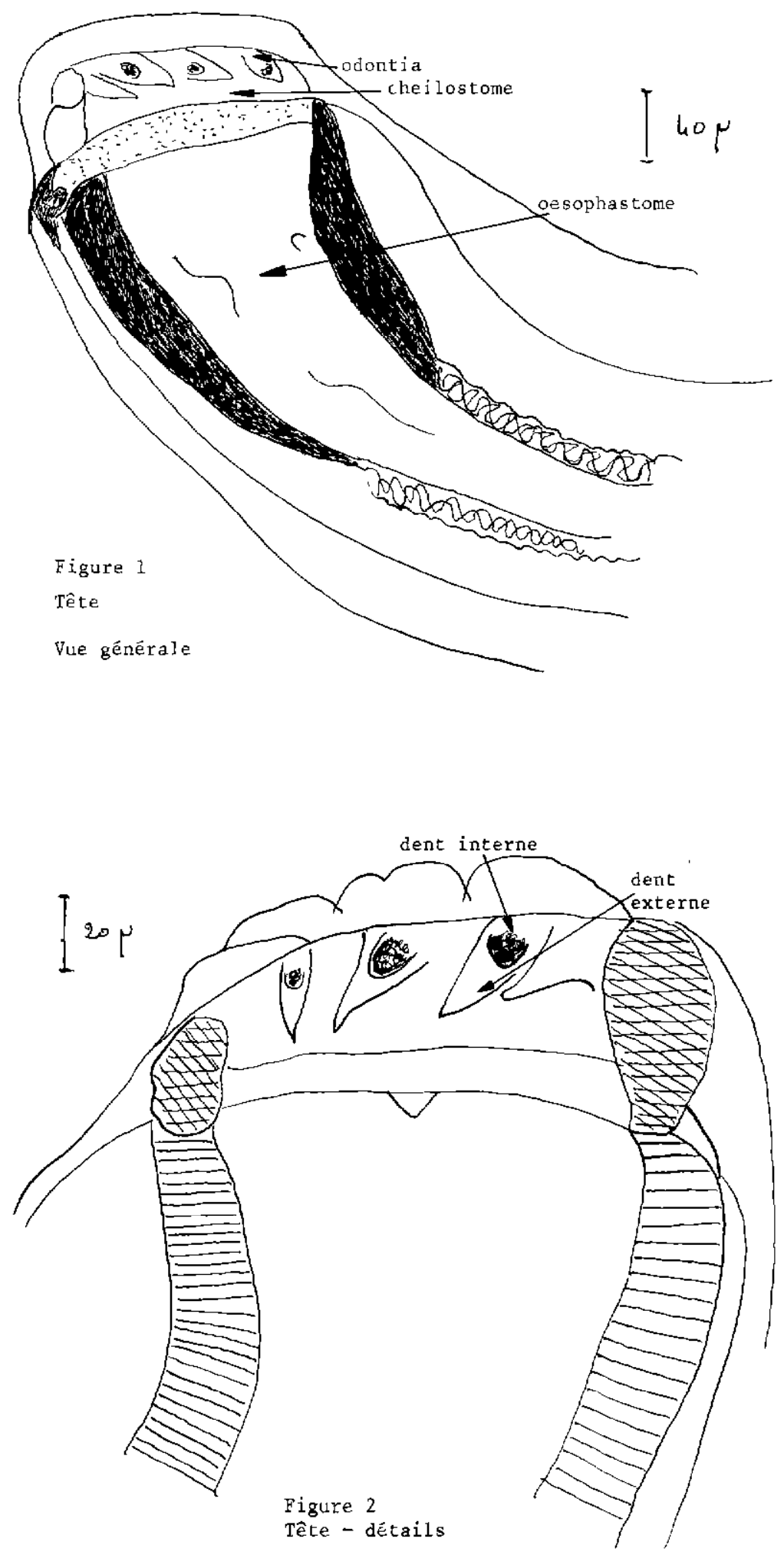
Chaque paire d'odontia subventrales, latérales et subdorsales comprend une dent externe bien visible et une dent interne très fine (fig. 2) qui est. en réalité, un tubercule de chitine attaché à la dent externe. Ce détail a échappé aux premiers observateurs et les descriptions de RAILLIET (19), LANE (12) et WARE (25) ne le mentionnent pas.

Les dents subdorsales de la $4^{e}$, les plus petites et les moins apparentes, sont fortement écartées l'une de l'autre. Les odontia de la $3^{e}$ paire sont massives. Les ventrales sont très rapprochées et cachées en partie par les odontia de la $2^{\mathbf{c}}$ paire.

Le rebord buccal, en avant des dents, est muni d'éléments chitineux pointus, difficiles à mettre en évidence, que LANE (12) a assimilé à «a primitive or degenerate corona radiata $»$.

La cavité qui fait suite à la bouche est divisée en deux parties inégales :

- la cavité buccale proprement dite ou Cheilostome (fig. 1), courte, large de $160-180 \mu$, plus haute ventralement $(60-70 \mu)$ que dorsalement (26-30 $\mu$ ). Son bord interne, en zone médio-ventrale, porte un plateau coupant réduit ;

- le tunnel asophagien ou Oesophastome (fig. 1), séparé du Cheilostome par une formation circulaire assez peu accentuée. Il a la forme d'un enorme entonnoir, à parois épaisses, long de 240-320 $\mu$ et large de 160-198 $\mu$. A l'intérieur et, en position symétrique, prennent place 3 lancettes, 2 du côté ventral et 1 du côté dorsal.

L'œsophage, long de 800 à $940 \mu$, se rétrécit au niveau de l'anneau nerveux, puis se dilate de nouveau en massue (260-280 $\mu)$. L'intestin qui le prolonge est large, irrégulier, rempli d'une matière gris-brunâtre constituée par du sang digéré et par des débris de cellules épithéliales. A la jonction des 2 organes, on note la présence de 3 valves, comme chez tous les Agriostomum.

Déirides et anneau nerveux sont à peu près au même niveau, à 545-595 $\mu$ de l'extrémité antérieure du ver.

La longueur et la largeur des mâles sont assez constantes : $9,8-10,8 \times 500 \mu$. L'ouverture orale est large de $120 \mu$.

La bourse caudale, relativement étroite, est soutenue par (fig. 3) :

- des côtes ventrales : nées d'un tronc commun, elles sont épaisses, droites et dirigées antérieurement ;

- des côtes latérales issues également d'un même tronc. Elles sont dirigées postérieurement.

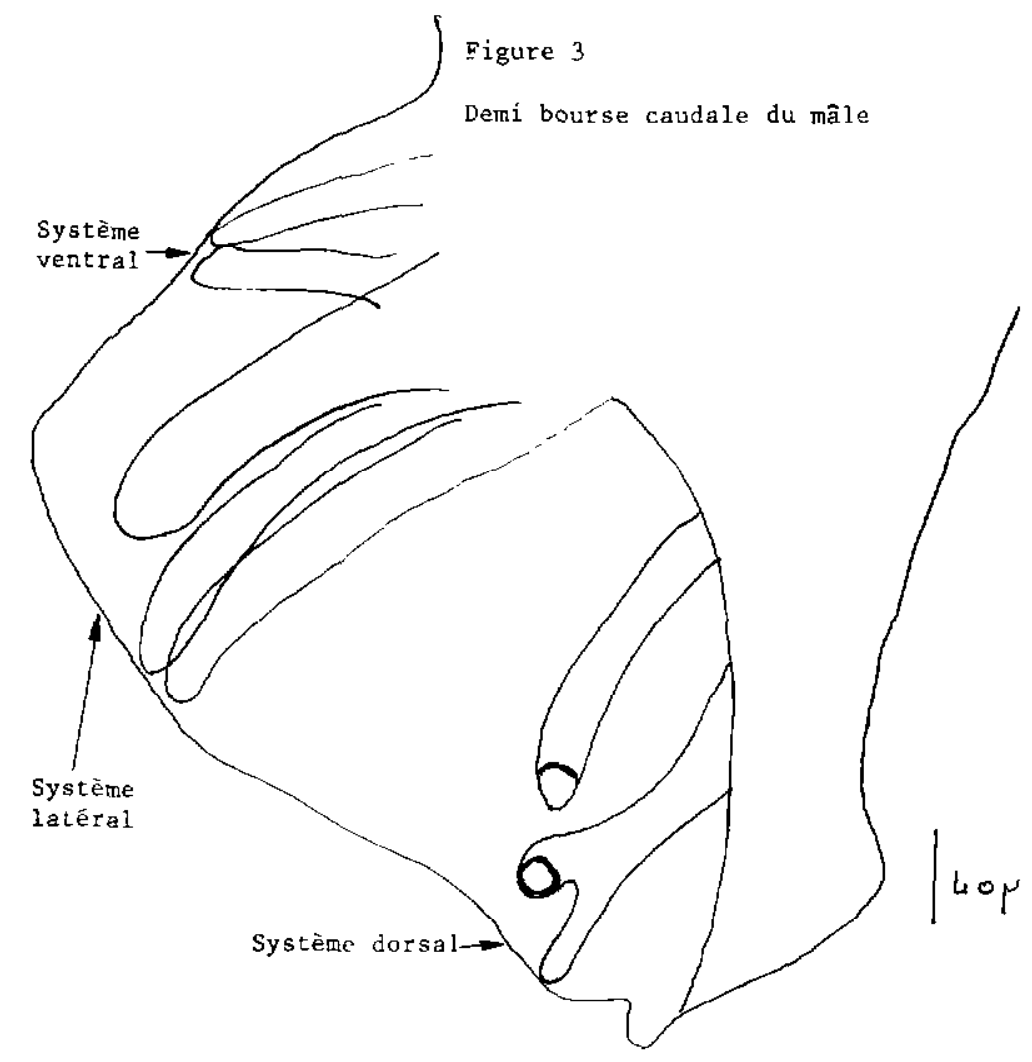



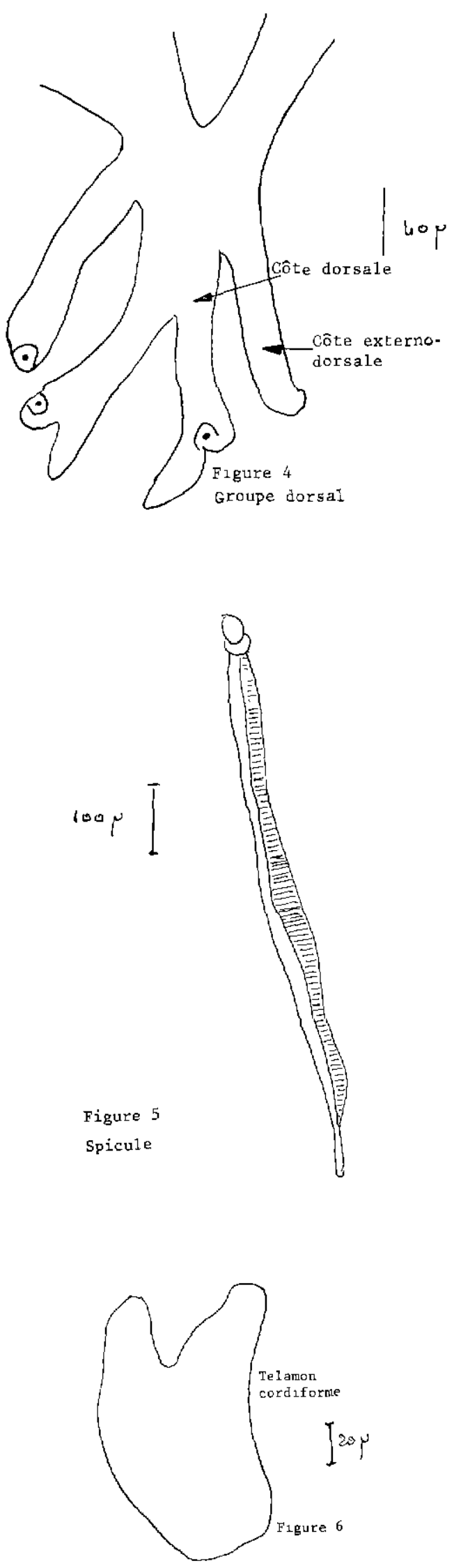

L'antéro-latérale, courte et arrondie à son extrémité, est nettement séparée de la médio-latérale et de la postéro-Iatérale, côtes juxtaposées et beaucoup plus longues que la précédente ;

- le groupe dorsal (fig. 4) :

- Une côte dorsale médiane bifurquée à son extrémité, chaque branche étant bidigitée, la digitation externe est petite et ronde, la digitation externe fine et pointue.

- Une côte externo-dorsale, forte et irrégulière qui se sépare du tronc commun avec la dorsale vers son tiers supérieur et qui se termine bien au-delà de la bifurcation de la dorsale, au niveau de sa digitation externe. Les spicules (fig. 5) qui mesurent de 827 à $850 \mu$ (moyenne, $838,4 \mu$ ) sont égaux, rectilignes et ailés sur presque toute leur longueur, sauf à l'extrémité distale qui se termine en pointe mousse. Ils sont maintenus par un télamon $(119 \times 74 \mu)$ cordiforme (fig. 6) prolongé par deux branches qui enserrent le bord externe des spicules. Il existe des papilles prébursales.

Le cône génital porte 2 papilles latérales dorsales (fig. 7), disposition que l'on retrouve chez la plupart des espèces appartenant au genre Agriostomum (18).

La femelle est plus longue $(13,1-13,6 \mathrm{~mm})$ et plus large $(600-640 \mu)$ que le mâle. La queue se termine en pointe aiguë légèrement recourbée (fig. 8). L'anus est à 125-160 $\mu$ de l'extrémité caudale et la vulve à $380-420 \mu$. Ces distances sont inférieures à celles indiquées par RAILLIET $(19,20)$, REYES (21) et WARE (25) *, ce qui n'est guère étonnant, car, chez beaucoup de Nématodes, elles sont susceptibles de varier dans des limites assez larges. La vulve donne accès à un vagin qui se prolonge par l'ovojecteur d'où partent un utérus antérieur et un utérus postérieur qui, très rapidement, se retourne vers l'avant.

Les œufs éllipsoïdes, à parois minces (fig. 9), sont segmentés au moment de la ponte. Ils mesurent 140-160 × 76-84 H (moyenne, $159,7 \times 79 \mu$ ).

Les Agriostomum comprennent actuellement 6 espèces que l'on peut séparer de la manière suivante :

1. Une fente ventrale. Côte externo-dorsale se terminant au niveau de la digitation externe

(*) Mais égales à celles de FERNANDES et GIOVANNONI (8). 

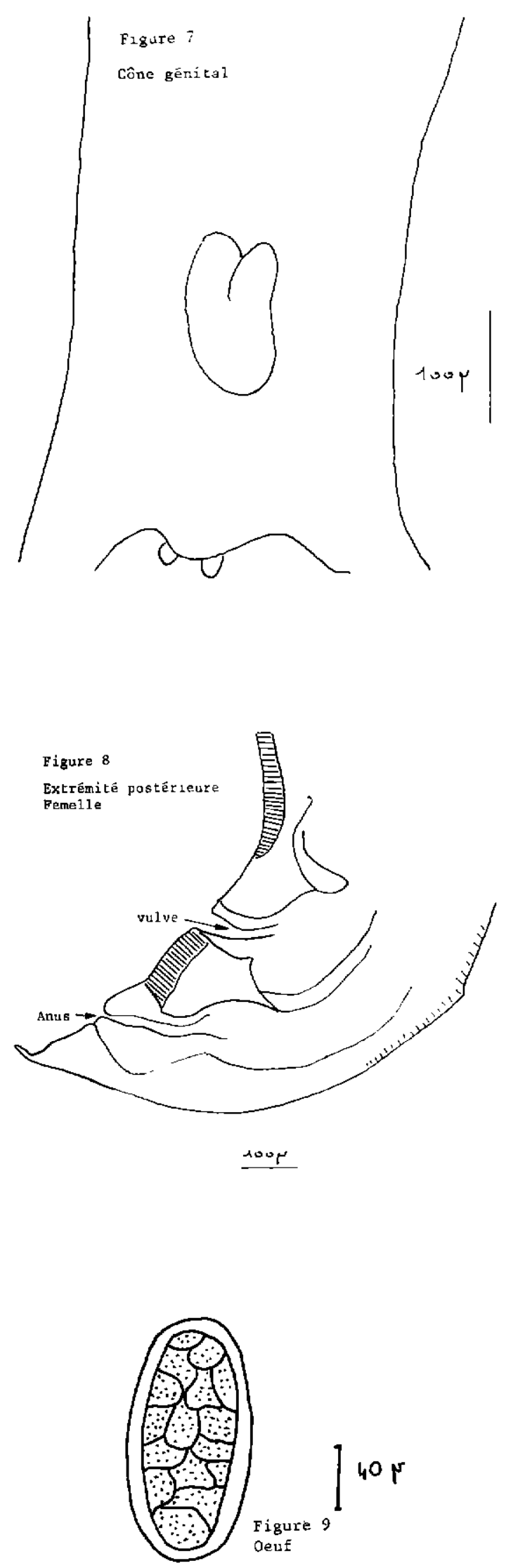

de la dorsale. Dent interne de chaque paire réduite. Spicules de $840-870 \mu$. Oeufs de 125 $192 \times 60-92 \mu$. Parasite des Ruminants domestiques : Agriostomum vryburgi Railliet, 1902.

2. Pas de fente ventrale. Côte externodorsale se terminant au niveau de la bifurcation de la dorsale ou légèrement au-delà. Dent interne de chaque paire bien marquée. Spicules de plus de $1 \mathrm{~mm}$. Oeufs de $64-88 \times 39-52 \mu$. Parasites des Bovidés sauvages africains.

2.1. Chaque paire d'odontia possède une dent externe et une dent interne de longueur égale :

- pas de dents dorso-latérales. Spicules de plus de $2 \mathrm{~mm}$. Agriostomum monnigi Ogden, 1965 ;

- dents dorso-latérales présentes. Spicules de $1,5 \mathrm{~mm}$. Agriostomum equidentatum Mönnig, 1929.

2.2. Dent externe de chaque paire plus longue que la dent interne.

- Taille moyenne (mâle $8-10 \mathrm{~mm}$; femelle 11-(4 mm) :

- spicules de 0,95-1,06 mm. Agriostomum gorgonis Le Roux, 1929 ;

- spicules de 1,47 mm. Agriostomum gorgonis var. congolensis Diaouré, 1964.

- Grande taille (mâle, 12,5-14 mm ; femelle, 14,5-17 mm). Spicules de 1,15-1,28 mm. Dent interne de chaque paire un peu plus longue que la dent homologue d'Agriostomum gorgonis. Agriostomum cursoni Monnig, 1932.

L'Agriostome découvert dans le tractus digestif des bovins de la Louila, par ses caractères anatomiques et ses dimensions, correspond donc bien à l'espèce Agriostomum vryburgi.

\section{COMMENTAIRES}

I. Agriostomum vryburgi est considéré classiquement comme un parasite du bétail - Zébu et Buffle - dans le Sud-Est asiatique. Il a été rencontré, en effet, aux Indes (2, 17, 25), en Indonésie (12, 19, 20, 24), au Vietnam (5), dans le Sud-Ouest de la Chine (23), en Malaisie $(6,7)$ et aux Philippines (21).

De plus, à la faveur des brassages de population animale, il a été introduit récemment au Brésil dans l'Etat de Parana $(3,8)$. 
En Afrique, jusqu'à présent, les Agriostomes étaient rigoureusement inféodés aux Bovidés sauvages: Damalisque, Bubale, Gnou, Oryx, Kob, Grand Koudou, Springbuck... (1, 4, 9, 10, $13,14,15,16,18)$.

L'existence d'Agriostomum vryburgi au Congo montre que les Bovidés domestiques n'en sont pas exempts.

Comme il a été dit plus haut, ce Nématode est nouveau pour l'Afrique.

Il est frappant de constater que l'aire de répartition géographique des Agriostomes correspond, en général, à un type de climat bien déterminé (II, 21) : il s'agit de régions humides où les précipitations annuelles sont supérieures à un mètre, avec une saison sèche de courte durée ( 3 à 4 mois au maximum).

2. La localisation des Agriostomes n'est pas constante et ils sont capables de vivre en différents points de l'intestin :

- Agriostomum equidentatum : intestin grêle (14) ; côlon $(15,16)$.

- Agriostomum gorgonis : iléon (13) ; côlon

- Agriostomum cursoni : intestin grêle $(15,16)$ duodénum, caecum, côlon $(9,10)$.

- Agriostomum vryburgi: duodénum (3, 8, $19,20,21)$; côlon $(5,6,7)$.

Certains ont conclu un peu hâtivement que les localisations antérieures (duodénum) étaient plus dangereuses que les localisations postérieures. En réalité, même dans la partie distale de l'intestin, plusieurs parasites (les Oesophagostomes larvaires notamment) peuvent avoir un rôle pathogène important et il n'y a aucune raison pour que les Agriostomes se comportent différemment.

3. Le rôle pathogène de ces parasites est encore mal connu. LE ROUX (13) estime que les Agriostomes - comme tous les Ancylostomidae et singulièrement les Bunostomes - sont de gros suceurs de sang. Grâce à leurs dents, ils s'implantent dans la muqueuse intestinale et, lorsqu'ils lâchent prise pour se fixer ailleurs, la blessure qu'ils ont provoquée laisse sourdre une goutte de sang, d'où des hémorragies. Dans le côlon de l'un des animaux abattus, il existait, en effet, un piqueté hémorragique dense, chaque tache représentant le point d'implantation d'un ver.
Il en résulte une anémie $(13,19,20)$ d'autant plus profonde que le Nématode est souvent associé à d'autres Helminthes: Bunostomum dans la région antérieure de l'intestin, Oesophagostomum dans la région postérieure.

Au Ranch de la Louila, l'association Oesophagostomes-Agriostomes est vraisemblablement à l'origine de l'importante mortalité observée en septembre 1974, époque de soudure alimentaire, aggravée cette année-là par une longue période de sécheresse qui a fortement réduit les ressources alimentaires disponibles.

4. Quelle en est l'origine ? En République populaire du Congo, des animaux en provenance du Congo-Kinshasa, du Cameroun et du Tchad ont été importés à plusieurs reprises. Or, dans ces contrées, l'agriostomose des bovidés domestiques est inconnue. En outre, aucune introduction de zébus asiatiques n'a été faite à ce jour.

Comme il s'agissait d'un lot de vaches N'Dama achetées au Sénégal en 1969-1970, on peut penser que les animaux étaient infestés au départ. Là encore, l'agriostomose n'a jamais été observée dans l'Ouest de l'Afrique. Toutefois, dans ce pays, il a été procédé à l'achat de quelques producteurs d'origine asiatique. Une contamination accidentelle a-t-elle pu se produire ? Dans l'état actuel de nos connaissances, il est bien difficile de répondre par l'affirmative.

D'une façon plus générale, pour pallier ces inconvénients, l'importation dans un pays d'animaux provenant d'une autre région, soit pour le peuplement, soit en vue d'une amélioration zootechnique, doit être effectuée avec le plus grand soin. Le contrôle doit concerner non seulement les maladies contagieuses, bactériennes et virales, mais encore les affections parasitaires, externes et internes. Sinon, on risque d'introduire des parasites dont le pays d'accueil est dépourvu. C'est le cas des Philippines où Agriostomum vryburgi a pénétré en même temps que des zébus indiens (Red Shindi, Ongole...) acquis par des organismes publics ou privés avant et après la seconde guerre mondiale et dont le contrôle parasitologique n'a pas été effectué (21).

Dans la littérature, on peut citer bien d'autres exemples et les règlements à l'jmportation des animaux domestiques et sauvages devraient en tenir compte. 


\section{CONCLUSIONS}

Au cours d'une enquête effectuée au Ranch de la Louila en décembre 1974, de nombreux parasites ont été recueillis à l'autopsie dans le tractus digestif de bovins N'Dama importés du Sénégal quelques années auparavant. Dans un lot d'Oesophagostomes, ont été découverts un certain nombre d'Agriostomes appartenant à l'espèce Agriostomum vryburgi Railliet, 1902 qui est redécrite.

C'est la première fois que ce Nématode du Sud-Est asiatique et d'Amérique du Sud est rencontré en Afrique dans l'intestin des Bovidés domestiques.
Il occasionne une forte anémie et, quand il est associé à des Bunostomes ou à des Oesophagostomes, ce qui est souvent le cas, il peut provoquer une mortalité quelquefois élevée.

La présence au Congo d'Agriostomum vryburgi, parasite jusqu'ici inconnu en Afrique équatoriale et dont la provenance ne peut être fixée de façon certaine, pose, cependant, le problème des importations de bétail. Lorsque l'on introduit dans un pays des bovins achetés dans une autre région, il est absolument nécessaire de rechercher un éventuel parasitisme interne et, s'il existe, de le faire disparaître à l'aide de traitements appropriés.

\section{SUMMARY}

Agriostomum pryburgi Railliet, 1902, a new cattle parasite in Popular Congo Republic

The authors point out the presence of Agriostomum vryburgi Railliet, 1902 in the colon of N'Dama cattle imported some years ago from Senegal and reared in Louila Ranch (Popular Congo Republic).

The Nematode, usually parasite of cattle and Buffalo in Brasil and Southeast Asia, is mentionned for the first time in Afrıca. Its origin is unknown.

The authors describe again the parasite and give some informations on its Jocalizations and pathological effects.

They emphasize the necessity of carefully examıning for internal parasites imported cattle.

\section{RESUMEN}

Agriostomum vryburgi Railliet, 1902, nuevo parásito de los bovinos domésticos de la República popular de Congo

Por la primera vez en Africa se señala la presencia de Agriostomum vryburgı Railliet, 1902 en el tracto digestivo de bovinos NDama importados de Senegal.

Se discute a propósito de la introducción posıble de dicho parásito en la República popular de Congo mediante ganado importado.

\section{BIBLIOGRAPHIE}

1. BAYLIS (H. A.). Records of some parasitic worms from the Belgian Congo. Ann. Mag. na Hist., Sér. II., $1939,3: 625-629$.

2. BHOPALE (K. K.), JOSHI (S. C.) et KAMALAPUR (S. K.). Occurrence of Agriostomum vryburgi Railliet, 1902 in an Indian Buffalo (Bos bubalis) in M. P. Orissa, Vet. J., 1971, 6 (2): 44-45.

3. COSTA (H. M.) et FREITAS (M. G.). Um raro parasito de Bovinos : Agriostomum vryburgi Railliet, 1902 (Nematoda-Strongyloidea). Archos Esc. Vet., B. Horizonte, 1963, 15: 147-151.

4. DIAOURE (A.). Strongylides parasites de Mammifères au Congo-Brazzaville. Annls. Parasit. hum. comp., 1964, 39 (3) : 246-249.

5. DROZDZ (J.) et MALCEWSKI (A.). Endoparasites et maladies parasitaires des animaux domestiques au Vietnam. Lab. Parasit. Acad. Sci., Varsovie, 1967: $110-112$.
6. EUZEBY (J.). Rep. Rome, F. A. O., 1956, No 493, $52 \mathrm{p}$.

7. EUZEBY (J.). Helmınthes du bétail et du porc dans la Fédération de Malaya. Rev. Elev. Méd. vét. Pay's trop., 1957, 10 (I) : 15-23.

8. FERNANDES (B. F.) et GIOVANNONI (M.) Ocorrencia de Agriostomum vryburgi Railliet, 1902 no Parana. Revta Esc. Agron. vet. Parana, 1966, 2 (1) : 113-I16.

9 GRABER (M.), DOUTRE (M.), FINELLE (P.), KERAVEC (J.), DUCROZ (G.) et MOKOTONGAR (P.). Les Helminthes de quelques Artiodactyles sauvages appartenant aux familles des Bovidés et des Suidés. Ces Mammfères, en République du Tchad, sont-ils des réservoirs de parasites pour les animaux domestiques vivant à leur contact ? Rev. Elev. Méd. vét. Pays trop., 1964, 17 (3) : 377-421. 
10. GRABER (M.). Rapport annuel Laboratorre de Farcha 1967. V. Pathologie de la Faune sauvage du Tchad. Premiers résultats d'enquêtes. N'Djaména, 1968, $157 \mathrm{p}$.

11. GRABER (M.). Enquête parasitologique concernant les ranches de la Louila et de la Louboulou - Niari - République populaire du Congo (Brazzaville). Rapport, Lyon, Ecole Nationale Vétérinaire, $1975,39 \mathrm{p}$.

12. LANE (C.). Some Strongylata. Parasitology, 1923, 15 (4) : 348-364.

13. LE ROUX (P. L.). On a Hookworm, Agriostomum gorgonis, sp. nov., from the blue wildebeest (Gorgon taurmus) of Transvaal. 15th Rep. Dir. vet. Servs., S. Afr., $1929: 481-491$.

14. MONNIG (H. O.). Agriostomum equidentatum, n. sp., a Hookworm of the Springbuck. 15th Rep. Dir. vet. Servs., S. Afr., 1929: 311-316.

15. MONNIG (H. O.). The genus Agriostomum with a description of Agriostomum cursoni. J. S. Afr. vet. med. Ass., 1932, 3 (1) : 16-21.

16. MONNIG (H. O.). Wild Antelopes as carriers of Nematode parasites of domestic ruminants. Onderstepoort $J$. vet. Sci. anim. Ind., 1933, 1 (1) : 77-92.

17. MUDALIAR (S. V.). Agriostomum vryburgi Railliet, 1902. Indian vet. $J ., 1942,19$ (3): 125-128.
18. OGDEN (C. G.). A new species of Hookworm, Agriostomum monnigi (Nematoda) from the Gemsbok, Oryx gazella. Ann. Mag. nat. Hist., Sér. 13, 1965, $8(93 / 94): 569-573$.

19. RAILLIET (A.). Sur quelques Sclérostomiens parasites des Ruminants et des Porcs. C. r. Séanc. Soc. Biol., 1902, 54 : 107-110.

20. RAILLIET (A.) et HENRY (A.). Sur les Esophagostomes des Ruminants. Bull. Soc. Path. exot., 1913, 6 (7): 506-511.

21. REYES (P. V.). Agriostomum vryburgi Railliet, 1902 from cattle in the Philippines. Philipp. J. Anim. Ind., 1963, 24 (1/4) : 57-61.

22. ROTH (H. H.) et DALCHOW (W.). Untersuchungen uber den Wurmbefall von Antilopen in Rhodesien. $Z$. angew. Zool., 1967, 54 (2) : 203-226.

23. SHU-CHING (W.), WEN-CHEN (Y.) et SHAOSUN (S.). A survey of the Helmuaths of the domestic animals in South-Western China. Acta zool, sin., 1965,17 (4) : 373-382.

24. SMIT (H. J.) et NOTOSOFDIRO (R.). Eenige Strongyliden onzer Huisdieren. Ned. Bl. Diergeneesl., $1923,35(2 / 3)$ : 191-198.

25. WARE (F. W.). Two uncommon parasites of cattle. J. comp. Poth. Ther., 1925, 38 (2) : 83-85. 